\title{
A NEW STATISTICAL RECONSTRUCTION METHOD FOR THE COMPUTED TOMOGRAPHY USING AN X-RAY TUBE WITH FLYING FOCAL SPOT
}

\author{
Robert Cierniak $^{1, *}$, Piotr Pluta ${ }^{1}$, Marek Waligóra ${ }^{2}$, Zdzisław Szymański ${ }^{3,4}$, \\ Konrad Grzanek ${ }^{5,4}$, Filip Pałka ${ }^{6}$,Vincenzo Piuri ${ }^{7}$ \\ ${ }^{1}$ Department of Intelligent Computer Systems, Czestochowa University of Technology, \\ Armii Krajowej 36, 42-200 Czesstochowa, Poland \\ ${ }^{2}$ Clinical Diagnostic Imaging Department, University of Opole, Opole, Poland \\ ${ }^{3}$ Management Department, University of Social Sciences, Lodz, Poland \\ ${ }^{4}$ Clark University, Worcester, MA 01610, USA \\ ${ }^{5}$ Institute of Information Technology, University of Social Sciences, Lodz, Poland \\ ${ }^{6}$ Faculty of Computer Science and Telecommunications, \\ Cracow University of Technology Warszawska 24, 31-155 Krakow, Poland \\ ${ }^{7}$ University of Milan, Department of Computer Science, Milan, Italy \\ *E-mail: robert.cierniak@pcz.pl
}

Submitted: 7th December 2020; Accepted: 9th August 2021

\begin{abstract}
This paper presents a new image reconstruction method for spiral cone- beam tomography scanners in which an X-ray tube with a flying focal spot is used. The method is based on principles related to the statistical model-based iterative reconstruction (MBIR) methodology. The proposed approach is a continuous-to-continuous data model approach, and the forward model is formulated as a shift-invariant system. This allows for avoiding a nutating reconstruction-based approach, e.g. the advanced single slice rebinning methodology (ASSR) that is usually applied in computed tomography (CT) scanners with X-ray tubes with a flying focal spot. In turn, the proposed approach allows for significantly accelerating the reconstruction processing and, generally, for greatly simplifying the entire reconstruction procedure. Additionally, it improves the quality of the reconstructed images in comparison to the traditional algorithms, as confirmed by extensive simulations. It is worth noting that the main purpose of introducing statistical reconstruction methods to medical CT scanners is the reduction of the impact of measurement noise on the quality of tomography images and, consequently, the dose reduction of X-ray radiation absorbed by a patient. A series of computer simulations followed by doctor's assessments have been performed, which indicate how great a reduction of the absorbed dose can be achieved using the reconstruction approach presented here.
\end{abstract}

Keywords: computed tomography, iterative statistical reconstruction method, flying focal spot 


\section{Introduction}

Despite the long history of medical computed tomography, the search for new designs of CT scanners still continues. At the beginning of the 21th century, the spiral scanner with an X-ray tube with a flying focal spot (FFS) $[1,2,3]$ has been introduced. This new technique aims at increasing the sampling density of the integral lines in the reconstruction planes and the density of simultaneously acquired slices in the longitudinal direction. This technique is realized in multidetector row CT (MDCT) scanners, but it allows for viewby-view deflections of the focal spot in the rotational $\alpha$-direction $(\alpha \mathrm{FFS})$ and/or in the longitudinal $z$-direction $(z F F S)$. Thanks to this characteristics, the quality of the reconstructed images can be improved, mainly by decreasing the influence of the aliasing effect in the reconstruction plane and in the $z$-direction, and by reducing the windmill artifacts. Obviously, efficient and effective FFS implementation needs new reconstruction methods that allow for directly using the measurements obtained from scanners. In practice, manufacturers decided primarily to modify the adaptive multiple plane reconstruction (AMPR) method for this purpose, (for details see, e.g. [4]). The AMPR technique is a nutating reconstruction method and, specifically, is an evolution of the advanced single slice rebinning (ASSR) algorithm [5, 6, 7]. Generally, nutating methods have several serious drawbacks, in particular equi-spaced resolution of the slices in $z$-direction is very difficult to obtain due to the constant change in the position of successive reconstruction planes. Another very significant problem is the limited ability to suppress noise, due to the linear form of the filters that are used. These drawbacks do not allow for using these methods in CT systems that aim to reduce the dose of $\mathrm{X}$-ray radiation absorbed by patients during examinations. Recent research in the area of X-ray computed tomography, including our own investigations, is mostly focused on addressing this challenge, because of the extremely harmful effect that CT examinations have on human health [8]. New approaches capable of reducing this impact are essential for the future existence of this popular, cheap, and effective image diagnostic technique. On the other hand, nowadays, the most interesting research directions in the area of
CT are statistical reconstruction approaches, especially those belonging to the model-based iterative reconstruction (MBIR) class of methods [9, 10]. Some commercial solutions have been developed by performing the reconstruction process in an iterative way to suppress noise in the obtained images. In this strategy, a probabilistic model of the individual measurements is taken into account and the reconstruction problem is reformulated into an optimization one. Unfortunately, the MBIR methods, which are used commercially (based on the iterative coordinate descent (ICD) algorithm [11, 12, 13]), have some serious drawbacks. The computational complexity of the problem is approximately proportional to $I^{4}$, where $I$ is the image resolution. The iterative reconstruction procedure based on this approach requires simultaneous calculations for all the voxels in the range of the reconstructed 3D image. Moreover, the size of the forward model matrix $\mathbf{A}$ is extremely large and it has to be calculated online. The reconstruction problem used in these approaches is also extremely ill-conditioned. This methodology is a method based on the discrete-todiscrete (D-D) data model. All those drawbacks can be reduced by using an approach based on a continuous-to-continuous (C-C) data model. In previous papers, we have proposed how to formulate the reconstruction problem based on the C-C data model that is consistent with ML methodology for scanners with parallel geometry [14], fanbeam scanners [15], and spiral cone-beam scanners $[16,17]$. Our conceptual approach has some significant advantages over the ones based on the DD model. First of all, in our method, the forward model is formulated as a shift invariant system, which allows for the use of FFT algorithms in the most computationally demanding parts of the reconstruction algorithm (realization of the 2D convolutions in the frequency domain). Furthermore, we can pre-calculate the model matrix (coefficients), i.e., we can generate it before our reconstruction algorithm starts. Finally, the reconstruction process can be carried out in only one plane in 2D space, thus greatly simplifying the reconstruction problem, and every slice of the body can be obtained separately. Our approach also outperforms the D-D method already at the level of problem formulation, regarding the better condition number [18]. In this paper, we present our innovative method based on statistical reconstruction for CT scanners with a fly- 
ing focal spot, which uses spiral cone-beam projections directly, similar to FDK-type algorithms [19].

\section{Geometry of the Spiral CT Scan- ner with Flying Focal Spot}

In our research, we have considered real CT scanners with flying focal spot. A general view of the spiral system analyzed, with its fundamental geometrical parameters, is shown in Figure 1.

In principle, the movement of the measuring system in this type of scanner does not differ from commonly used devices. The only difference comes down to the use of a variable focus X-ray tube, the so-called flying focal spot, with all that that entails. That is why we can describe the movement of the measuring system, and the topology of the X-ray system used, separately.

\subsection{Design of the measuring system}

As usual, in spiral scanners, the X-ray source (X-ray tube) and the screen with detectors rotates around the $z$-axis while a patient, lying on the table, translates in the direction of this axis (see e.g. [7]). The detailed geometry of the movement of the scanner, with fundamental trigonometric parameters is depicted in Figures 2, 3 and 4.

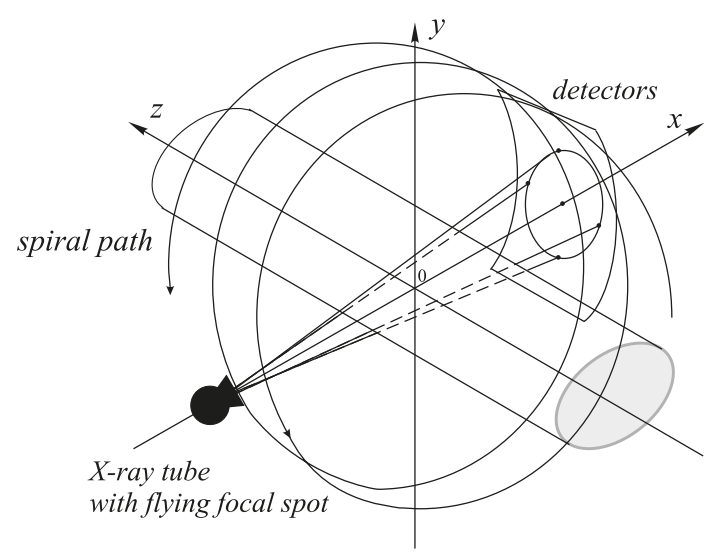

Figure 2. A perspective view of the measurement system

It is quite easy to see that while the measurement system rotates around the $z$ axis the view angle $\alpha$ is changed, proportionally with the progress of the sliding movement of this system. This means that the focus of the X-ray tube, whose position is defined by the parameter $z_{0}$, moves along the $z$-axis according to the following formula (under the assumption that $z_{0}=0$ at an angle $\alpha=0$ ):

$$
z_{0}=\lambda \frac{\alpha}{2 \pi}
$$

where: $\lambda$ is a constant that defines the table increment per full revolution of the tube.

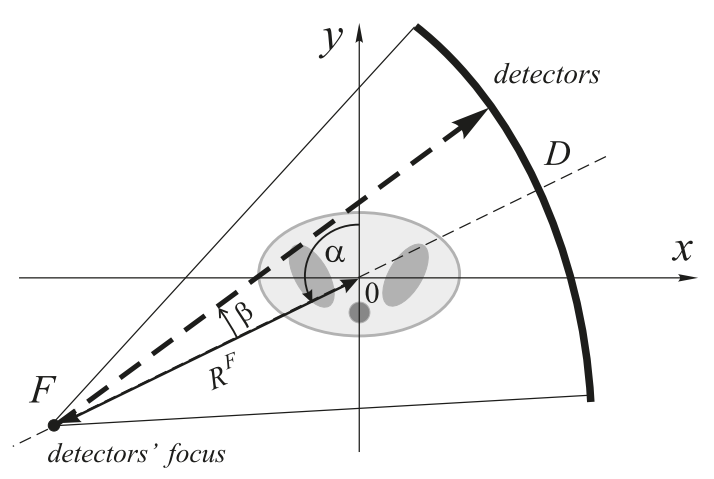

Figure 3. The view in a reconstruction plane

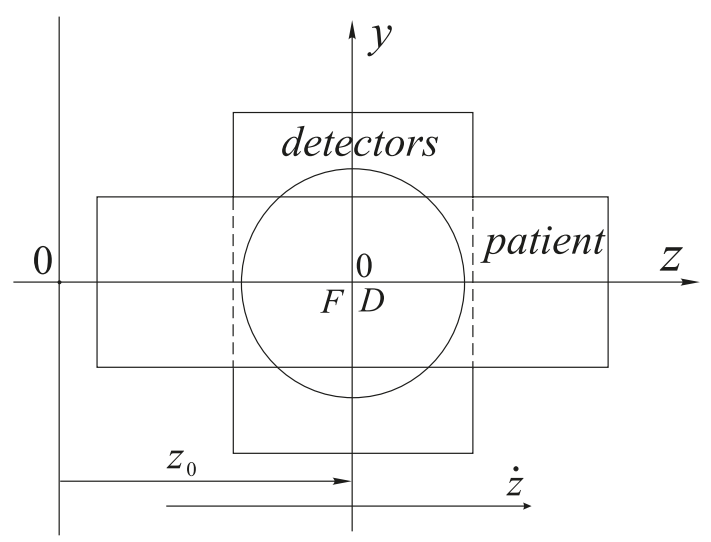

Figure 4. The view in the plane along the $z$ axis

Individual measurements are performed at given angles $\alpha_{\theta}$; where this value is incremented by a constant value $\Delta_{\alpha}(\theta=0,1, \ldots)$. These measurements are carried out using detectors fixed on the screen, and in this way projections $p$ are obtained. It is worth noting that an integral line constituting the projection run from a given focus spot $F$ on the screen to a given detector identified by the pair of indexes $\psi$ (the number of a column in the detector matrix: $\psi=-(\Psi-1) / 2, \ldots, 0, \ldots,(\Psi-1) / 2$, and $\Psi$ is an odd number of detectors in each array) and $k$ (the number of a row in the detector array: $k=1,2, \ldots, K$, and $K$ is an even number of rows). As is shown in Figures 3 and 4, each such 


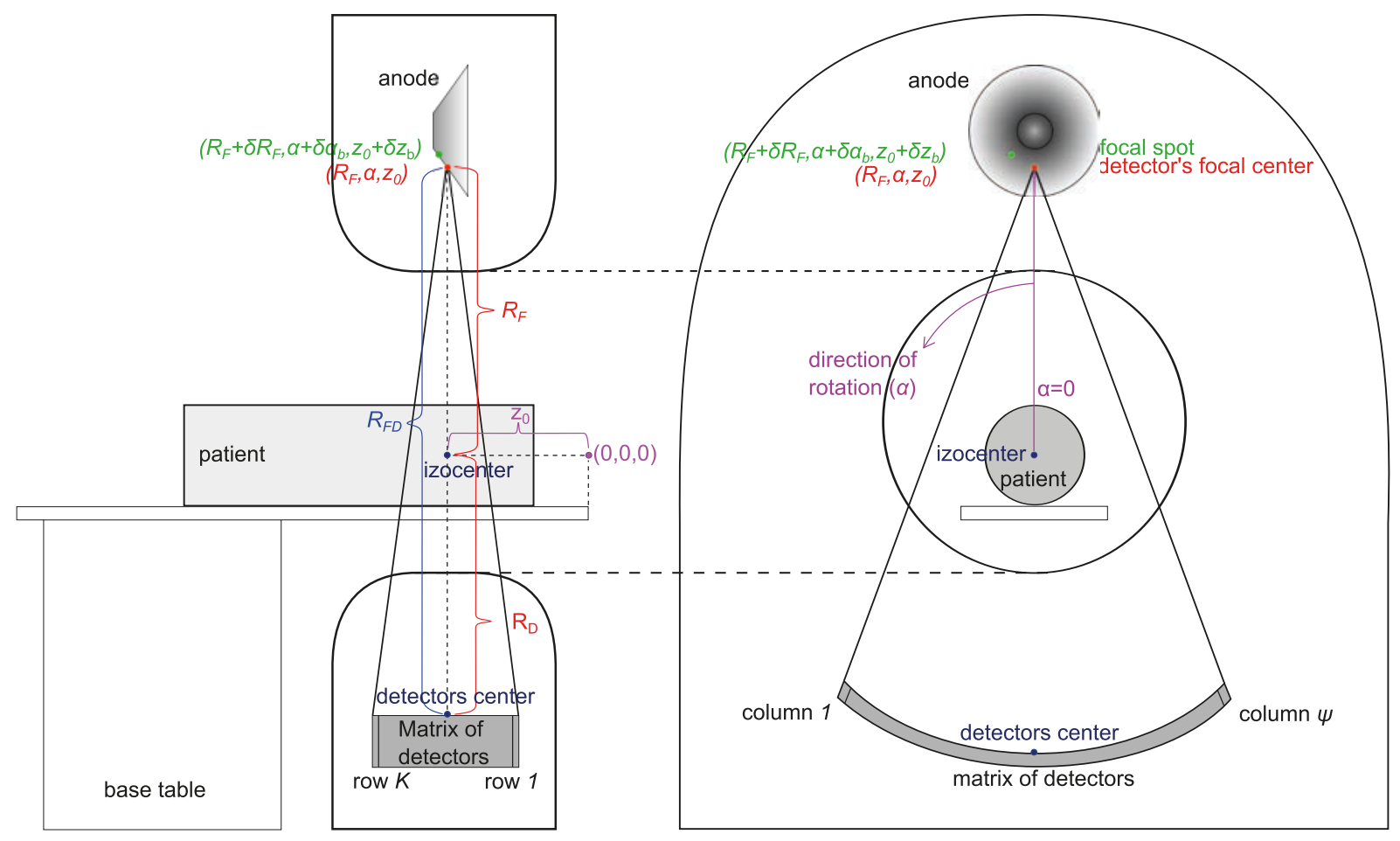

Figure 1. Geometry of the measurement system of the analyzed scanner: in the $z$-direction (A); in the reconstruction plane (B)

integral line is described by an angle $\beta_{\psi}$ (the angle between a particular ray and the line aligning with the isocenter and a given focal center) and by a distance $\dot{z}_{k}$ (the $z$-coordinate relative to the current position of the rotating measurement system). Therefore, any projection can be written as a function $p\left(\alpha_{\theta}, \beta_{\psi}, \dot{z}_{k}\right)$.

Other parameters describing the geometry of this measurement system are the detector focus center - isocenter distance $R^{F}$ and the normal detector focus center - detector center distance $R^{F D}$.

\subsection{Flying focal spot system}

The flying focal spot technique makes use of a special construction of X-ray tube, where it is possible to deflect the electron beam (by means of an electric field) before it hits the anode of the tube. This mechanism allows for view-by-view deflections of the focal spot for X-rays emitted from that anode. The topology of the focal spots on the anode differs depending on the given scanner (an Xray tube was used). A view of the practical solution, regarding placement of focal spots on the anode, is depicted in Figure 5.
In this technique, the focal spot is switched periodically between these places on the anode of the $\mathrm{X}$-ray tube according to an established procedure. Thanks to this mechanism, several working modes of the scanner are practicable, namely: the flying focal spot in the $\alpha$-direction (so called $\alpha F F S$ ), the flying focal spot in the $z$-direction (so-called $z \mathrm{FFS}$ ), and in both these directions together (see also [2]). Generally, the flying focal spot in the $\alpha$-direction aims to increase the resolution of the sampling of the integral lines in the reconstruction plane, and in the $z$-direction it aims to improve the resulting resolution of the reconstruction planes in the longitudinal direction [3]. This latter effect causes a decrease of the aliasing in the $z$-direction, and consequently, prevents the production so-called windmill artifacts. Views of both these modes of resolution improvement are presented, together with fundamental parameters, in the Figures 6 and 7 (For details regarding the method for determining the geometrical parameters of a scanner with a flying focal spot see also e.g. [2] and [4]).

It should be clearly noted that changing the focal spot during the measurement process also affects the parameters of the projection system, i.e. 
the focus-isocenter distance $R^{F}$, the rotation angle $\alpha$ and the $z$ position of the focal spot. The small deviations of these parameters will be marked as: $\delta R$, $\delta \alpha$ and $\delta \dot{z}$, respectively. Because we possess projections that were made using only two focal spots, i.e. those assigned in Figures 6 and 7 as $A$ and $B$, we present the deviations of these parameters in Table 1 for these two locations only.
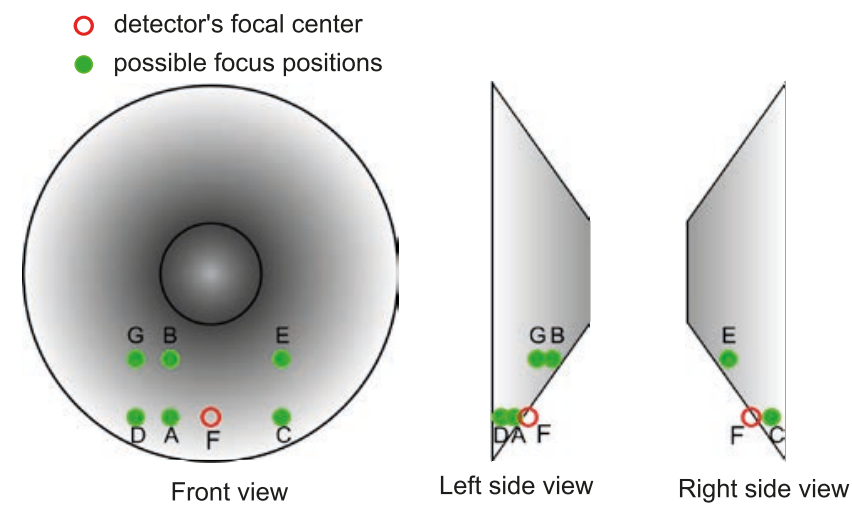

Figure 5. Topology of the focal spots on the anode: orientation in the reconstruction plane and both of the side views

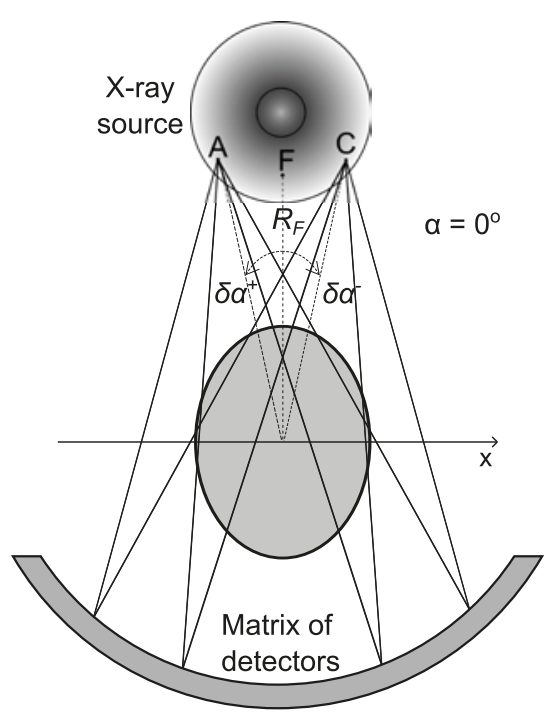

Figure 6. View of the flying focal spot in the $\alpha$-direction

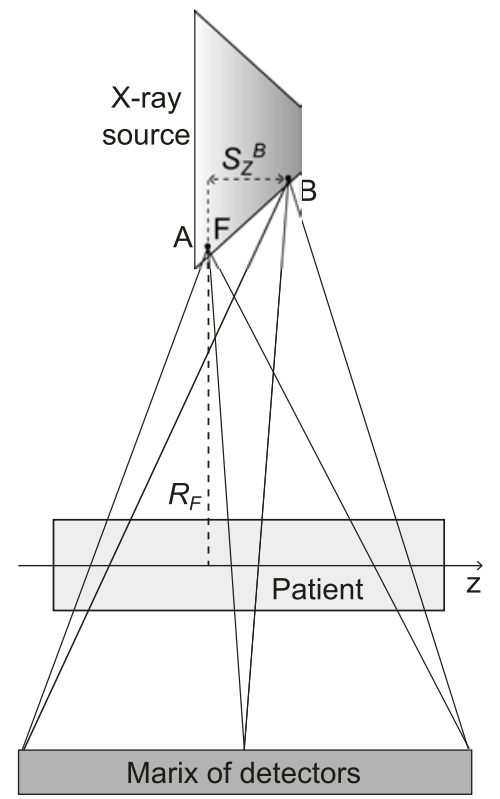

Figure 7. View of the flying focal spot in the $z$-direction

Table 1. Combination of $\alpha$-direction and $z$-direction deviations

\begin{tabular}{c|c|c|c}
\hline \hline Parameter/Focal spot & $R^{f s}$ & $\alpha$ & $\dot{z}$ \\
\hline $\mathrm{A}$ & 0 & $\delta \alpha^{A}$ & 0 \\
\hline $\mathrm{B}$ & $\delta R^{B}$ & $\delta \alpha^{B}$ & $\delta \dot{z}^{B}$ \\
\hline \hline
\end{tabular}

One can summarize the values in Table 1 as follows: in the case of focus position A, only an increment in the $\alpha$-direction is specified, and in the case of focus position $\mathrm{B}$, all three deviations have to be taken into account. Using these values, it is possible now to determine the adjusted angles of rotation [2], as follows

$$
\alpha^{f s}=\alpha+\delta \alpha^{f s},
$$

where: $f s$ is an indication of the given focal spot $(A$ or $B$ ).

In turn, for focal spot $B$ the adjusted position of the detector with index $k$ can be established using the following formula,

$$
\dot{z}_{k}^{B}=\dot{z}_{k}+\delta \dot{z}^{B},
$$

where $\dot{z}_{k}$ means the positions of the given detector $k$.

And finally, also for both focal spots, the nominal focus-isocenter distance has to be increased, as follows 


$$
R^{f s}=\sqrt{\left(R^{F}+\delta R^{f s}\right)^{2}+\left(\left(R^{F}+\delta R^{f s}\right) \tan \delta \alpha^{f s}\right)^{2}} .
$$

It is easy to establish relations (2)-(4) considering Figure 8 , which describes schematically the geometrical conditions regarding the deviations of $\alpha$, $R^{F}$ and $\dot{z}$, according to the non-central focal spot placement.
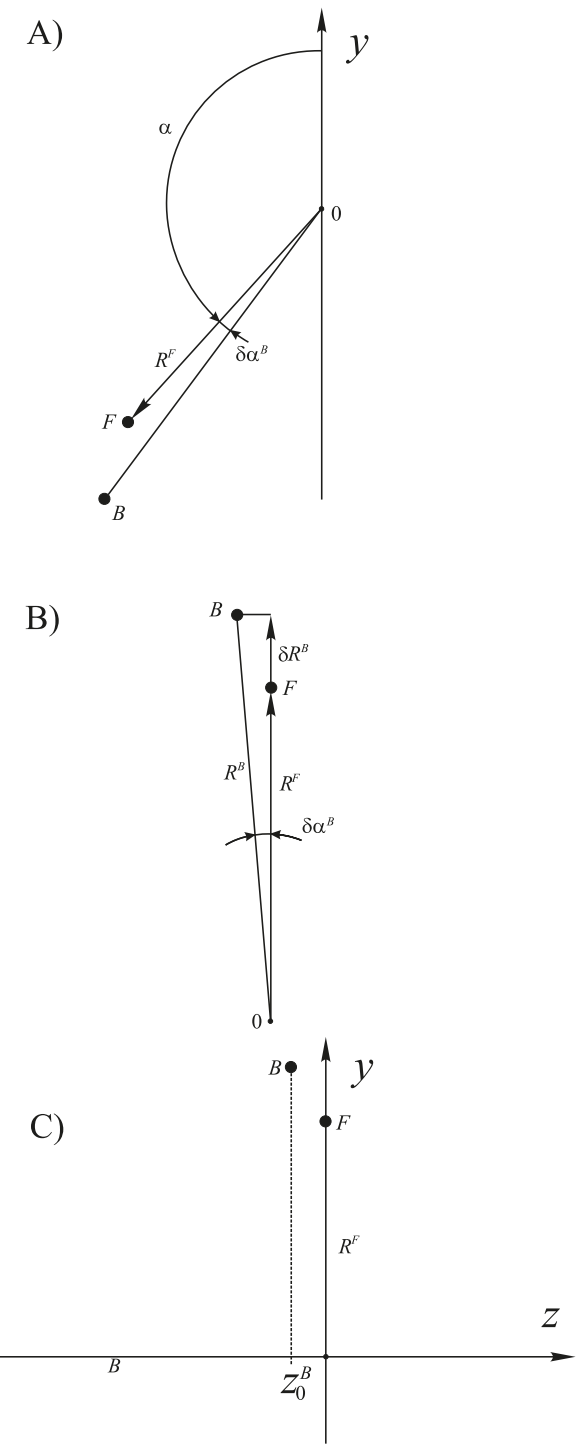

Figure 8. Geometry of the deviation of the projection parameters: $\alpha(\mathrm{A}), R^{F}$ (B) and $\dot{z}(\mathrm{C})$

It should be noted that for both focal spot positions, the integral lines are not equi-angularly distributed, and the real parameter of each ray, i.e. the angle $\beta_{\psi}^{f s}$ between a particular ray and the line aligning with the isocenter and a given focal spot, has to be calculated separately [2], as follows:

$$
\beta_{\psi}^{f s}=\measuredangle\left(-\mathbf{f}^{f s}, \mathbf{d}_{\psi}-\mathbf{f}^{f s}\right),
$$

where at the position of the X-ray tube at $\alpha=$ $-P i / 2$ :

$$
\mathbf{f}^{f s}=\left[\begin{array}{c}
x_{f s} \\
y_{f s}
\end{array}\right]=\left[\begin{array}{l}
R^{f s}+\delta R^{f s} \\
\left(R^{F}+\delta R^{f s}\right) \tan \delta \alpha^{f s}
\end{array}\right],
$$

describes the coordinates of the focus positions, and

$$
\mathbf{d}_{\psi}=\left[\begin{array}{l}
x_{d} \\
y_{d}
\end{array}\right]=\left[\begin{array}{l}
R^{F}-R^{F D} \cos \beta_{\psi}^{F} \\
-R^{F D} \sin \beta_{\psi}^{F}
\end{array}\right]
$$

describes the coordinates of the positions of the individual detectors, wherein the nominal angles $\beta_{\psi}^{F}$, in every fan beam, can be calculated based on the following relation:

$$
\beta_{\psi}^{F}=\left(\psi-\psi^{F}\right) \Delta_{\beta}^{F},
$$

where: $\psi^{F}$ is the index of the detector element aligning with the isocenter and the detectors focal center; $\Delta_{\beta}^{F}$ is the angular distance between any two detectors if the integral lines come from the detectors focus.

Having Eqs (6)-(7), it is possible to determine the values of the non eqi-angular distributed angles $\beta_{\psi}^{f s}$, following the relation:

$\beta_{\psi}^{f s}=\arcsin \frac{x_{f s}\left(y_{d}-y_{f s}\right)-\left(x_{d}-x_{f s}\right) y_{f s}}{\sqrt{x_{f s}^{2}+\left(x_{d}-x_{f s}\right)^{2}} \sqrt{y_{f s}^{2}+\left(y_{d}-y_{f s}\right)^{2}}}$.

A schematic depiction of the idea behind the determination of the real angles of the rays in the fans is shown in Figure 9.

The above considerations allow the calculation of the angles $\beta_{\psi}$ for a chosen focal spot position, particularly Eqs (6)- (9), and the equations presented earlier Eqs (2)-(4) for determining the adjusted angles of rotation for them, the adjusted positions of the detectors and the modified focusisocenter distance, respectively. Now, our method has the modifications of the basic parameters of the projections performed by the scanner ready to use for all possible placements of the focal spot. 


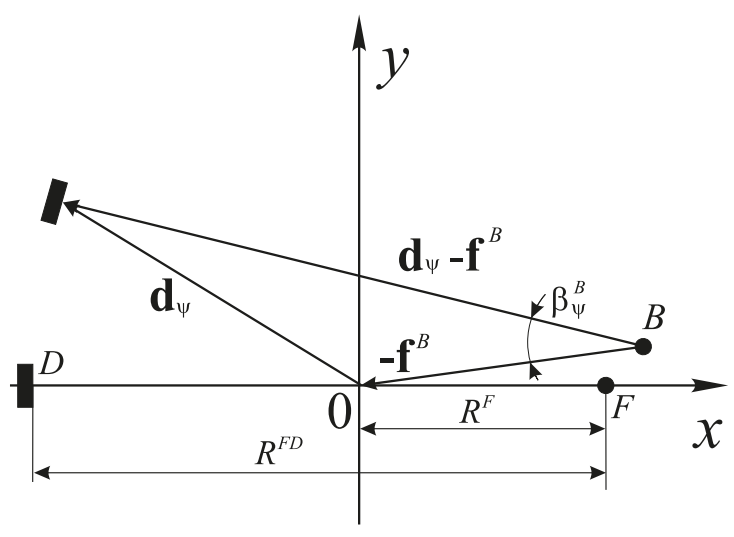

Figure 9. Geometry of the angles $\beta_{\psi}$ determination

\section{Statistical Reconstruction Algo- rithm}

Our proposed reconstruction method, for the case of the flying focal spot technique, is based on the maximum-likelihood (ML) estimation [9], [10]. Usually, the objective of this kind of approach to the reconstruction problem is formulated according to a discrete-to-discrete (D-D) data model. In opposition to that category of the methods, we propose an optimization formula that is consistent with the continuous-to-continuous (C-C) data model [20], as follows:

$$
\begin{gathered}
\mu_{\min }=\arg \min _{\mu}\left(\int _ { x } \int _ { y } \left(\int_{\bar{x}} \int_{\bar{y}} \mu(\bar{x}, \bar{y}) .\right.\right. \\
\left.\left.h_{\Delta x, \Delta y} d \bar{x} d \bar{y}-\tilde{\mu}(x, y)\right)^{2} d x d y\right),
\end{gathered}
$$

where $\tilde{\mu}(x, y)$ is the image obtained after the performance of a back-projection operation. If we take into account only projections from one focal spot position, then this image is described theoretically in the following way:

$$
\begin{gathered}
\tilde{\mu}^{f s}(x, y) \approx \int_{0}^{2 \pi} \int_{-\beta_{\max }}^{\beta_{\max }} p^{f s}\left(\beta, \alpha, z_{k}\right) \\
\text { CORR } \cdot \operatorname{int}_{L}(\Delta \beta) d \beta d \alpha,
\end{gathered}
$$

wherein $p^{f s}(\beta, \alpha, \dot{z})$ are projections carried out using a given focal spot in a spiral cone-beam scanner, and the coefficients $h_{\Delta i, \Delta j}$ can be determined according to the following formula:

$$
h_{\Delta x, \Delta y}=\int_{0}^{2 \pi} i n t(\Delta x \cos \alpha+\Delta y \sin \alpha) d \alpha,
$$

and $\operatorname{int}(\Delta s)$ is an interpolation function (we used the linear interpolation function).

It is worth underlining that the correction term present in Eq. (11) corresponds to the only correction to be done, and the reasoning for this is as follows: integral lines defining hypothetical fan-beams going through the reconstructed crosssection pass through almost the same tissues as rays from a cone-beam geometry having only one common point of intersection with the reconstruction plane. Because of this, we can derive the correction factor by using the following relation:

$$
C O R R=\frac{R^{F D}}{\sqrt{R^{F D^{2}}+z_{k}^{2}}},
$$

where: $z_{k}$ is the transverse position on the screen where a given ray is detected.

According to the discrete realization of the reconstruction method presented above, described by Eqs (10)-(13), it is feasible to present the iterative approach formulated by us, as follows:

$$
\begin{aligned}
& \mu_{\min }=\arg \min _{\mu}\left(\sum _ { i = 1 } ^ { I } \sum _ { j = 1 } ^ { I } \left(\sum_{\bar{i}}^{I} \sum_{\bar{j}}^{I}\right.\right. \\
&\left.\left.\mu\left(x_{\bar{i}}, y_{\bar{j}}\right) \cdot h_{\Delta i, \Delta j}-\tilde{\mu}\left(x_{i}, y_{j}\right)\right)^{2}\right),
\end{aligned}
$$

where:

$$
\tilde{\mu}^{f s}\left(x_{i}, y_{j}\right)=\Delta_{\alpha} \sum_{\theta} \dot{p}^{f s}\left(\beta_{i j}, \alpha_{\theta}, \dot{z}_{i j}\right),
$$

where it is necessary to use interpolation to obtain approximated projections at points $\beta_{i j}$ based on the measured projections $p^{f s}\left(\beta_{\eta}, \alpha_{\theta}, \dot{z}_{k}\right)$.

We can obtain these projections in the following way:

$$
\begin{aligned}
& \dot{p}^{f s}\left(\beta_{i j}, \alpha_{\theta}, \dot{z}_{i j}\right)= \\
& \sum_{k} \sum_{\eta} p^{f s}\left(\beta_{\eta}, \alpha_{\theta}, \dot{z}_{k}\right) \frac{R^{F D}}{\sqrt{R^{F D^{2}}+z_{k}^{2}}} . \\
& \quad \operatorname{int}_{\beta}\left(\beta_{i j}-\eta \Delta_{\beta}\right) \operatorname{int}_{z}\left(z_{i j}-k \Delta_{z}\right),
\end{aligned}
$$


where $\operatorname{int}_{\beta}(\Delta \beta)$ and $\operatorname{int}_{z}(\Delta z)$ are the linear interpolation functions:

$$
\operatorname{int}_{\beta}(\beta)=\left\{\begin{array}{lll}
\frac{1}{\Delta_{\beta}}\left(1-\frac{|\beta|}{\Delta_{\beta}}\right) & \text { for } & |\beta| \leq \Delta_{\beta} \\
0 & \text { for } & |\beta| \geq \Delta_{\beta}
\end{array},\right.
$$

and

$$
\operatorname{int}_{z}(z)=\left\{\begin{array}{lll}
\frac{1}{\Delta_{z}}\left(1-\frac{|z|}{\Delta_{z}}\right) & \text { for } & |z| \leq \Delta_{z} \\
0 & \text { for } & |z| \geq \Delta_{z}
\end{array} .\right.
$$

The coefficients $h_{\Delta i, \Delta j}$ are in this case calculated using the following formula:

$$
\begin{aligned}
& h_{\Delta i, \Delta j}=\Delta_{S}^{2} \Delta_{\alpha} \\
& \sum_{\psi=0}^{\Psi-1} \operatorname{int}\left(\Delta i \cos \psi \Delta_{\alpha}+\Delta j \sin \psi \Delta_{\alpha}\right),
\end{aligned}
$$

wherein $\operatorname{int}(\Delta s)$ is a form of the interpolation function exactly as that used in the back-projection operation, and we suppose $\Delta_{s}=R^{F} * \tan \Delta_{\beta}$.

It is a big advantage of the approach presented here that both the correction and the backprojection operations can be performed separately (in parallel) for every focal spot (in our case, for two focal spots). These images, in our case $\tilde{\mu}^{A}\left(x_{i}, y_{j}\right)$ and $\tilde{\mu}^{B}\left(x_{i}, y_{j}\right)$, we can add them directly, i.e.:

$$
\tilde{\mu}\left(x_{i}, y_{j}\right)=\frac{\tilde{\mu}^{A}\left(x_{i}, y_{j}\right)+\tilde{\mu}^{B}\left(x_{i}, y_{j}\right)}{2},
$$

and then perform the iterative reconstruction procedure accordingly, which is described by the formula (14). This is carried out using the sum of these two images, i.e. $\tilde{\mu}\left(x_{i}, y_{j}\right)$.

There are some technical problems associated with the back-projection operation, especially performing the interpolation, in the situation when the distribution of the integral lines is no longer equiangular, and this interpolation then has to be performed at a different resolution in every case.

The complete algorithm, that is proposed here, is described schematically in Figure 10. It is worth noting that in this scheme an FFT algorithm can be implemented to realize the convolutions in the frequency domain, and this significantly accelerates the necessary calculations.

\subsection{Iterative reconstruction procedure}

Formula (14) can be the basis for the formulation of many iterative approaches to the image reconstruction from projections problem, obviously consistent with ML methodology. In our approach, we used the Newton-Raphson method, because of its simplicity. In this case, the pixels in the reconstructed discrete image will take the following values, in an iterative way:

$$
\begin{array}{r}
\mu^{*(t+1)}\left(x_{i}, y_{j}\right)=\mu^{*(t)}\left(x_{i}, y_{j}\right)- \\
c \cdot \sum_{\bar{i}=1}^{I} \sum_{\bar{j}=1}^{I} e^{(t)}\left(x_{\bar{i}}, y_{\bar{j}}\right) h_{\Delta i, \Delta j},
\end{array}
$$

where: $c$ is a constant coefficient, $i=0, \ldots, I$ and $j=0, \ldots, I, I$ is the dimension of the reconstructed image, and

$$
e^{(t)}\left(x_{\bar{i}}, y_{\bar{j}}\right)=\sum_{i=1}^{I} \sum_{j=1}^{I} \mu^{(t)}\left(x_{i}, y_{j}\right) h_{\Delta i, \Delta j}-\tilde{\mu}\left(x_{\bar{i}}, y_{\bar{j}}\right) .
$$

In our method, every iteration of the reconstruction procedure is comprised of two steps described by relations (21) and (22) (the possible development of this approach is presented in [21]). It is fundamental for our conception that the convolutions from these relations can be changed to multiplications through transformation of the calculations in the frequency domain. Thanks to this idea, the computational complexity is decreased from an $I^{4}$ to a $\log _{2} I^{2}$ problem. Obviously, we have also to take into consideration the fact that in every iteration additional Fourier and inverse Fourier transformations have to be performed.

It should be emphasized that the iterative reconstruction procedure presented above is conducted for the joined image $\tilde{\mu}(x, y)$ expressed by Eq. (20), representing all focal spot positions used (in our case only two positions). This is absolutely contrary to the solution proposed in [22], where all integral lines from all focal spots are taken into account at the formulation of the objective in the reconstruction problem. 


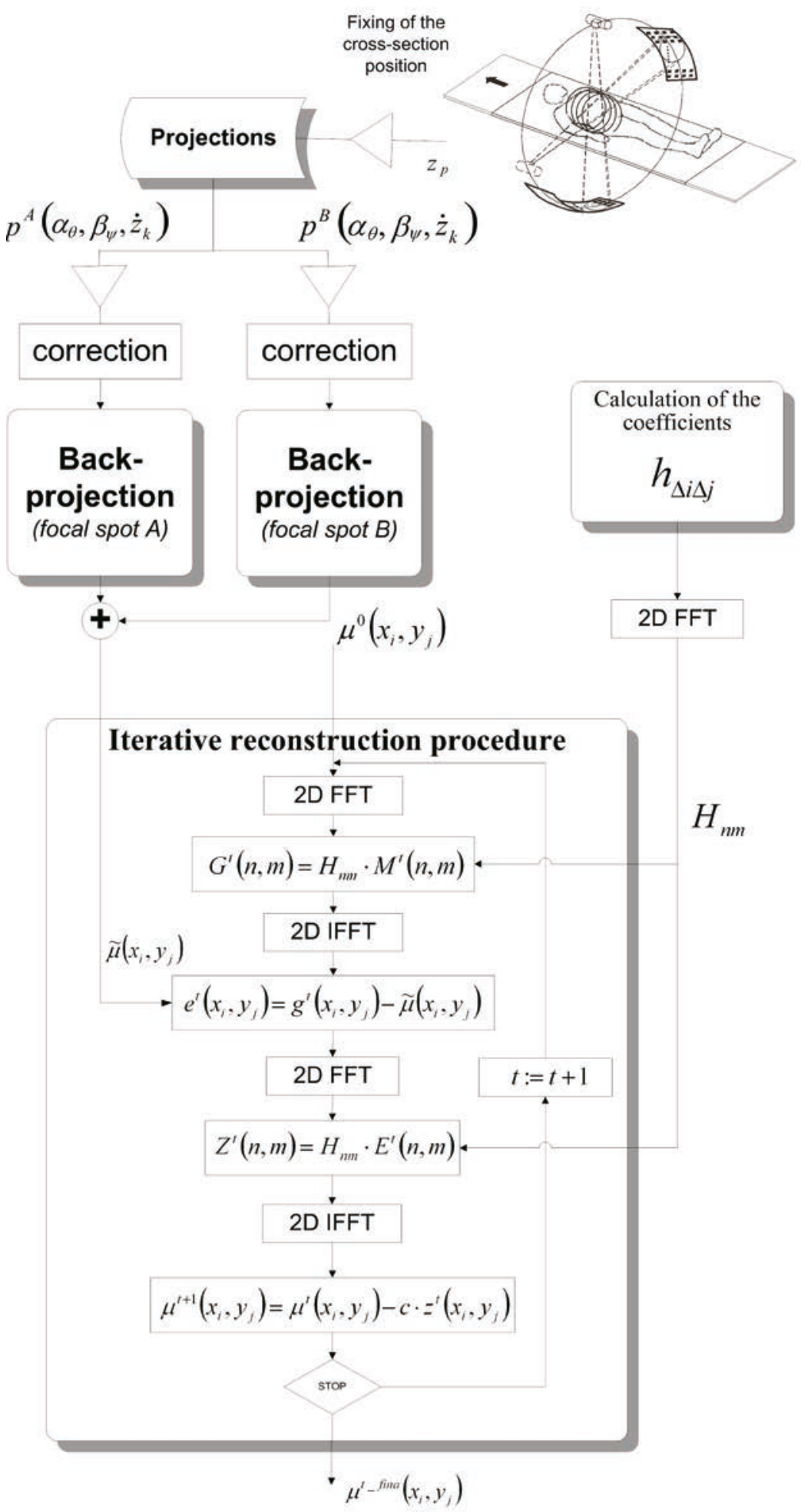

Figure 10. Diagram of the complete statistical reconstruction algorithm for scanner with the flying focal spot technique 


\section{Experimental Results}

In the experiments which we have conducted, we used measurements obtained from a medical scanner Somatom Definition AS+, Siemens AG, Forchheim, Germany. All projections were obtained using the helical mode, using the following scanner parameters: reference tube potential $120 \mathrm{kVp}$ and the tube current $200 \mathrm{mAs}, R^{F D}=$ $1085.6 \mathrm{~mm}, R^{F}=595 \mathrm{~mm}$, number of views per rotation $\Psi=1152$, number of detector rows 736 , number of detector columns 32 , the dimensions of the individual detectors were $1.09 \mathrm{~mm} \times 1.28 \mathrm{~mm}$. We have determined the size of the reconstructed image to be $512 \times 512$ pixels, at the real size of this image $360.1 \mathrm{~mm} \times 360.1 \mathrm{~mm}$

A complete set of coefficients representing the matrix $h_{\Delta i, \Delta j}$ was pre-calculated before the reconstruction process was started, and saved to the computer memory for the whole iterative reconstruction procedure (in order to accelerate these calculations, the coefficients were transformed into the frequency domain). Firstly, in every medical case, the images were obtained after the back-projection operations were determined (without any filtration) for all of the focal spot positions used, separately. However, before that all the individual projections used for the back-projection operation were corrected, according to relation (12). Then all these images were combined into only one single image, and in this way the referential signals in the optimization objective were established. Finally, an iterative reconstruction procedure was performed. As the starting point for this procedure a specially prepared result of an FBP reconstruction algorithm (using projections obtained from the focal spot position $A$ ) was set. It is worth underlining that our iterative reconstruction procedure was performed without any form of regularization term regarding the objective function from (14).

In our experiments, we carried out the calculations necessary to realize the iterative reconstruction using two hardware implementations, i.e. one based on a GPU (nVidia Titan V) and one using a CPU with 10 cores (Intel i9-7900X BOX/3800MHz processor). We present the results obtained using these two realizations in Table 2. A radiologist assessed that 7000 iterations are enough to provide an acceptable image from a diagnostic point of view.
Table 2. Comparison of the computation times for the realizations of the iterative reconstruction procedure used

\begin{tabular}{|c|c|c|}
\hline Nuber of iterations & GPU & CPU \\
\hline \hline 1000 & $850 \mathrm{~ms}$ & $705 \mathrm{~ms}$ \\
\hline 7000 & $7443 \mathrm{~ms}$ & $4932 \mathrm{~ms}$ \\
\hline 10000 & $9945 \mathrm{~ms}$ & $7045 \mathrm{~ms}$ \\
\hline \hline
\end{tabular}

From a practical point of view, the most important thing is to deliver CT images that are useful diagnostically, and of course without harming patients. It is quite difficult to determine the balance between these two opposing goals, pointing strictly to the best solution. We will try to show the possibilities for decreasing the dose absorbed by patients presenting with different forms of pathological changes. At the beginning, we compare the results obtained in the presence of a large pathological change by assessing the views of the reconstructed images in Figure 11, where a full-dose examination is used, and in Figure 12 where a quarterdose examination is considered. In both cases, Figures marked as (A) depict reconstructed images obtained using the traditional FDK algorithm with linear interpolation function and Shepp-Logan kernel, Figures (B) and (C) represent reconstructed images, where the iterative statistical method proposed in this paper is used, when only measurements performed using the first focal spot position were utilized (B), and when measurements from both focal spots were utilized (C).

The same form of comparison is presented in Figure 13 and Figure 14, but in the medical case where small pathological changes in the liver are present.

The results presented above were not representative enough, and so the next experiment had to objectify the results obtained. For this purpose, we used 47 images obtained using a full-dose, and the same 47 images using an X-ray dose reduced by a quarter, with and without any small pathological changes, which were earlier diagnosed based on the basis of full-dose images of the same slices. Additionally, we used images obtained using only the focal spot $A$ (extra dose reduction by half). The reconstructed images were then carefully assessed diagnostically by a radiologist, and he marked any lesions that he noticed in the $\mathrm{CT}$ images according 

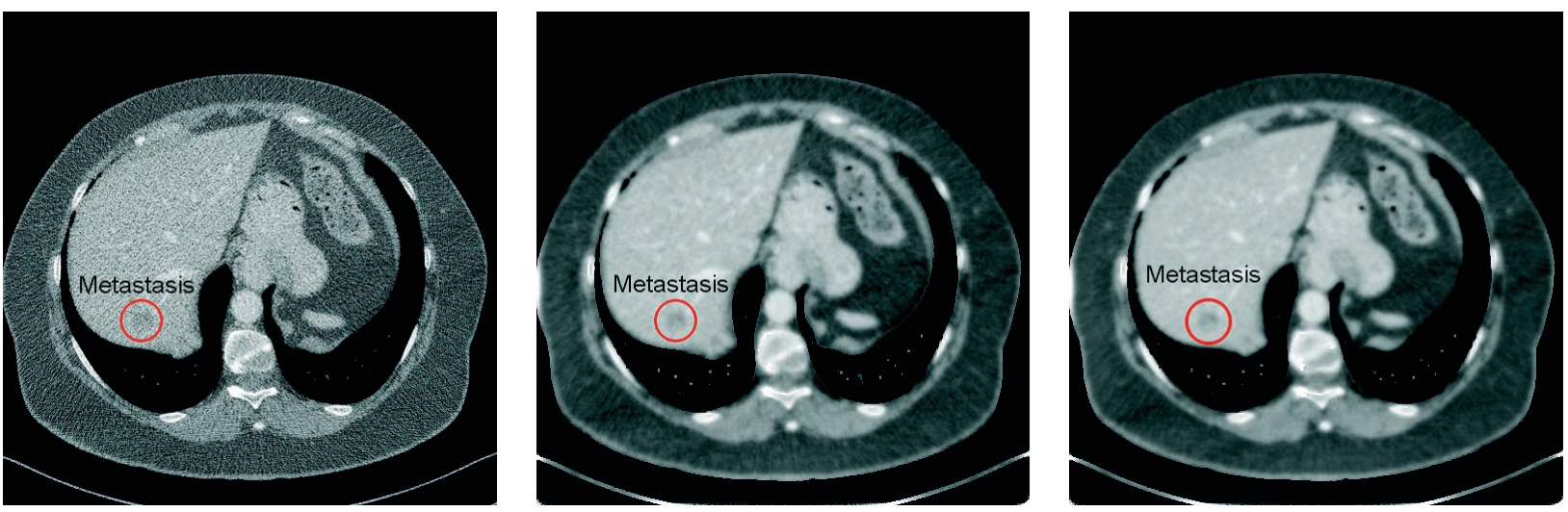

Figure 11. View of the reconstructed image in a case with a large pathological change in the liver, using full-dose projections with the application of: the traditional FDK algorithm (A); the iterative statistical method proposed in this paper (the first focal spot position) (B); the iterative statistical method proposed in this paper (both focal spot positions) (C).
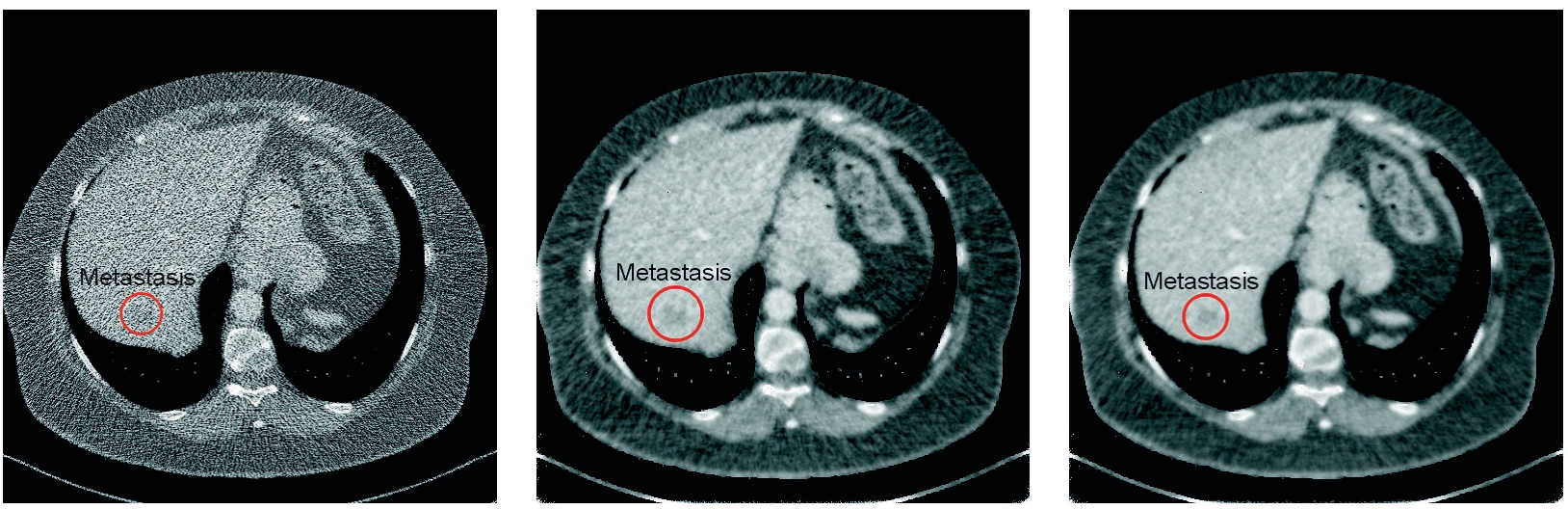

Figure 12. View of the reconstructed image in a case with a large pathological change in the liver, using a quarter-dose examination with the application of: the traditional FDK algorithm (A); the iterative statistical method proposed in this paper (the first focal spot position) (B); the iterative statistical method proposed in this paper (both focal spot positions) (C) 

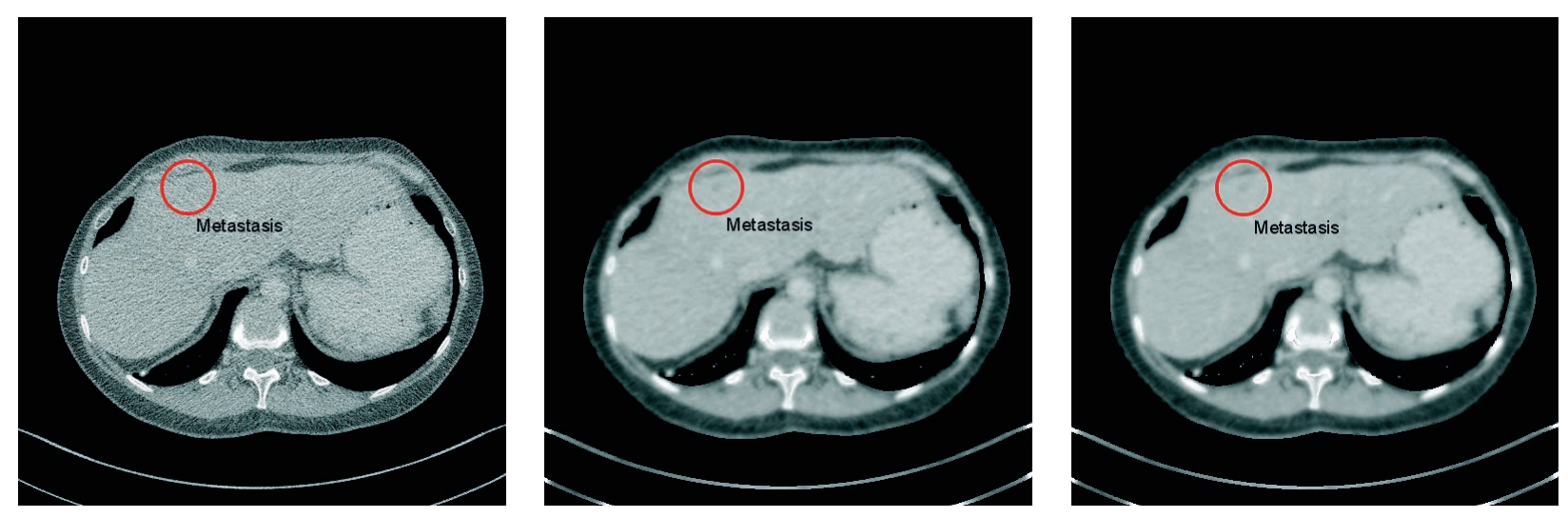

Figure 13. View of the reconstructed image in a case with small pathological changes in the liver, using full-dose projections with the application of: the traditional FDK algorithm (A); the iterative statistical method proposed in this paper (the first focal spot position) (B); the iterative statistical method proposed in this paper (both focal spot positions) (C).
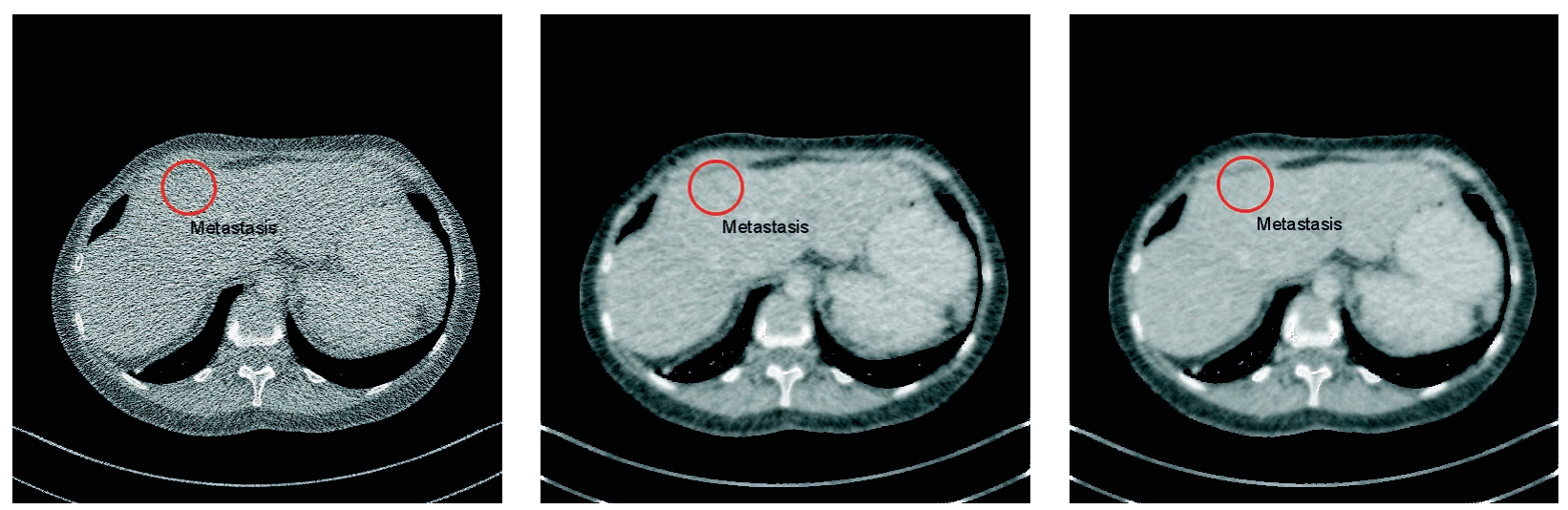

Figure 14. View of the reconstructed image in a case with small pathological changes in the liver, using a quarter-dose examination with the application of: the traditional FDK algorithm (A); the iterative statistical method proposed in this paper (the first focal spot position) (B); the iterative statistical method proposed in this paper (both focal spot positions) (C) 
to his subjective opinion. Tables 3, 4 and 5 show the scores obtained compared to the true diagnoses. That means that the table shows the numbers of true positive (TP) cases (i.e. at least one lesion correctly marked in a case with lesions), false negative (FN) cases (i.e. no lesions marked in a case that had lesions), true negative (TN) cases (i.e. no lesions marked in a case with no lesions) and false positive (FP) cases (i.e. at least one lesion marked in a case with no lesions). Note that if the assessment of a given case was ambiguous, then this case received a grade of 0.5 , and if a diagnostic evaluation was without any doubts, then this given case obtained a grade of 1 or 0 .

Table 3. Results of the diagnoses made by a radiologist using images obtained at a full dose of radiation (used the focal spots $A$ and $B$ ).

\begin{tabular}{|c||c|c|c|c|}
\hline Rec. method & TP & FN & TN & FN \\
\hline $\begin{array}{c}\text { Statistical } \\
\text { approach }\end{array}$ & 22 & 5 & 19.5 & 0.5 \\
\hline
\end{tabular}

Table 4. Results of the diagnoses by a radiologist using images obtained at a dose of radiation reduced by half (used only the focal spot $A$ ).

\begin{tabular}{|c||c|c|c|c|}
\hline Rec. method & TP & FP & TN & FN \\
\hline $\begin{array}{c}\text { Statistical } \\
\text { approach }\end{array}$ & 19 & 8 & 19.5 & 0.5 \\
\hline $\begin{array}{c}\text { FDK } \\
\text { algorithm }\end{array}$ & 15.5 & 11.5 & 19.5 & 0.5 \\
\hline
\end{tabular}

Table 5. Results of the diagnoses by a radiologist using images obtained at a dose of radiation reduced by a quarter.

\begin{tabular}{|c||c|c|c|c|}
\hline Rec. method & TP & FP & TN & FN \\
\hline $\begin{array}{c}\text { Statistical } \\
\text { approach } \\
(f s=A \text { and } B)\end{array}$ & 15.5 & 11.5 & 19.5 & 0.5 \\
\hline
\end{tabular}

Table 6. Results of the diagnoses by a radiologist using images obtained at a dose of radiation reduced eight times (a dose of radiation reduced by a quarter and used only the focal spot $A$ ).

\begin{tabular}{|c||c|c|c|c|}
\hline Rec. method & TP & FP & TN & FN \\
\hline $\begin{array}{c}\text { Statistical } \\
\text { approach } \\
(f s=A)\end{array}$ & 11 & 16 & 19.5 & 0.5 \\
\hline $\begin{array}{c}\text { FDK } \\
\text { algorithm } \\
(f s=A)\end{array}$ & 3 & 24 & 19.5 & 0.5 \\
\hline
\end{tabular}

The results shown in Tables 3-6 by the following metrics can be summarized: sensitivity, accuracy and $F_{1}$ score, according to the following definitions:

$$
\begin{gathered}
\text { sensitivity }=\frac{T P}{T P+F N}, \\
\text { accuracy }=\frac{T P+T N}{T P+F N+T N+F P},
\end{gathered}
$$

and

$$
F_{1} \text { score }=\frac{T P}{T P+\frac{1}{2}(F P+F N)} .
$$

Values of the above metrics for all cases from Tables 3-6 are presented in Table 7.

Table 7. The results of the diagnostic experiments expressed with selected metrics.

\begin{tabular}{|c||c|c|c|}
\hline $\begin{array}{c}\text { dose reduction/ } \\
\text { (reconst. method) }\end{array}$ & sens. & accur. & $F_{1}$ score \\
\hline $\begin{array}{c}\text { full dose } \\
\text { (stat. approach) }\end{array}$ & 0.815 & 0.883 & 0.889 \\
\hline $\begin{array}{c}\text { half dose } \\
\text { (stat. approach) }\end{array}$ & 0.704 & 0.819 & 0.817 \\
\hline $\begin{array}{c}\text { half dose } \\
\text { (FDK alg.) }\end{array}$ & 0.574 & 0.745 & 0.721 \\
\hline $\begin{array}{c}\text { quarter dose } \\
\text { (stat. approach) }\end{array}$ & 0.0574 & 0.744 & 0.0 .721 \\
\hline $\begin{array}{c}\text { fullose } \\
\text { (stat. approach) }\end{array}$ & 0.407 & 0.627 & 0.571 \\
\hline $\begin{array}{c}\text { fulldose } \\
\text { (FDK alg.) }\end{array}$ & 0.111 & 0.479 & 0.196 \\
\hline
\end{tabular}




\section{Conclusion}

An original complete statistical iterative reconstruction method that can be used in scanners with a flying focal spot has been presented here. Experiments conducted by us have proved that our reconstruction approach is relatively fast (approximately the same results were achieved for both hardware implementations: the iterative reconstruction procedure takes less than $7.5 \mathrm{~s}$, and in the case of the GPU, it is less than 5s), mainly thanks to the use of an FFT algorithm during the most demanding calculations in the iterative reconstruction procedure and thanks to efficient programming techniques. It should be emphasized that the computational complexity of our approach is proportional to $I^{2} \log _{2} I$, if the image resolution is assumed to be $I \times I$ pixels. Additionally, the iterative reconstruction procedure was implemented without introducing any additional regularization term, using only an early stopping regularization strategy, which enables a decrease of the time taken by the reconstruction process when compared to the referential approach proposed in [22]. It can be considered, from the practical point of view, that our method yields satisfactory results regarding the quality of the reconstructed images, with the dose absorbed by the patients being reduced even by a quarter. (It is worth emphasizing that we considered only diagnostically challenging medical cases with minor pathological changes). Unfortunately, it would be difficult to compare the results obtained by us with a referential nutating approach, because those methods give images that are not perpendicular to the $z$-axis, and it uses projections performed within a rotation angle of $180^{\circ}$, whereas in our method we can use all the projections from a full revolution of the scanner. Moreover, our method is very easy to implement and open to the use of different modes of focal spot modalities. Finally, and no less importantly, the price of the hardware needed for our method is relatively very low (it is about 5000 USD in both cases). Further research will be devoted to integrating computational intelligence methods into the approach presented here.

\section{Acknowledgement}

The authors thank Dr. Cynthia McCoullough and the American Association of Physicists in
Medicine for providing the Low-Dose CT Grand Challenge dataset.

This work was supported by The National Center for Research and Development in Poland (Research Project POIR.01.01.01-00-0463/17).

\section{References}

[1] P. Schardt, J. Deuringer, J. Fruedenberger, E. Hell, W. Knuüpfer, D. Mattern, M. Schild, New X-ray tube performance in computed tomography by introducing the rotating envelope tube technology, Medical Physics, vol. 31, 2004, pp. 2699-2706.

[2] T. Flohr, T. G., Stierstofer, K. Ulzhaimer, S. Ulzheimer, H. Bruder, A. N. Promak, C. H. McCollough, Image reconstruction and image quality evaluation for a 64-slice CT scanner with zflying focal spot, Medical Physics, vol. 32, 2005 , pp. 2536-2547.

[3] M. Kachelrieß, M. Knaup, C. Penssel, W. A. Kalender, Flying focal spot (FFS) in cone-beam CT, IEEE Transactions on Nuclear Science, vol. 53, 2006, pp. 1238-1247.

[4] Th. Flohr, K. Stierstofer, H. Bruder, J. Simon, A. Polacin, S. Schaller, Image reconstruction and image quality evaluation for a 16-slice CT scanner, Medical Physics, vol. 30, 2003, pp. 832-845.

[5] M. Kachelrieß, S. Schaller, W. A. Kalender, Advanced single-slice rebinning in cone-beam spiral CT, Medical Physics, vol. 27, 2000, pp.754-773.

[6] M. Kachelrieß; Th. Fuchs, S. Schaller, W. A. Kalender, Advanced single-slice rebinning for tilted spiral cone-beam CT, Medical Physics, vol. 28, 2001, pp.1033-1041.

[7] R. Cierniak, P. Pluta, A. Kaźmierczak, A practical statistical approach to the reconstruction problem using a single slice rebinning method, Journal of Artificial Intelligence and Soft Computing Research, vol. 10, 2021, pp. 137-149.

[8] J. D. Mathews et al., Cancer risk in 680 peope expose to computed tomography scans in childhood or adolescent: data inkage study of 11 million Australians, British Medical Journal, f2360, 2013, pp. 346-360.

[9] K. Sauer, C. Bouman, A local update strategy for iterative reconstruction from projections, IEEE Transactions on Signal Processing, vol. 41, 1993, pp. 534-548.

[10] C. Bouman, K. Sauer, A unified approach to statistical tomography using coordinate descent optimization. IEEE Transations on Image Processing, vol. 5, 1996, pp. 480-492. 
[11] J. -B. Thibault, C. A. Bouman, K. D. Sauer, J. Hsieh, A recursive filter noise reduction in statistical iterative tomographic imaging, Proc. of SPIE-IS\&T Symposium on Electronic Imaging Science and Technology-Computational Imaging, vol. 6065, 2006, pp. 15-19.

[12] J. -B Thibault, K. D. Sauer, C. A. Bouman, J. Hsieh, A three-dimensional statistical approach to improved image quality for multislice helical CT, Med. Phys., vol. 34, 2007, pp. 4526-4544.

[13] Y. Zhou, J.-B. Thibault, C.A. Bouman, J. Hsieh, J., K.D. Sauer, Fast model-based X-ray CT reconstruction using spatially non-homogeneous ICD optimization, IEEE Tranactions on Image Processing, vol. 20, 2011, pp. 161-175.

[14] R. Cierniak, A new approach to image reconstruction from projections problem using a recurrent neural network, International Journal of Applied Mathematics and Computer Science, vol. 183, 2008, pp. 147-157.

[15] R. Cierniak, New neural network algorithm for image reconstruction from fan-beam projections, Neurocomputing, vol. 72, 2009, pp. 3238-3244.

[16] R. Cierniak, A three-dimensional neural network based approach to the image reconstruction from projections problem, Lecture Notes in Artificial Intelligence, vol. 6113, 2010, pp.505-514.

[17] R. Cierniak, Analytical statistical reconstruction algorithm with the direct use of projections per- formed in spiral cone-beam scanners, In Proc. of the 5th International Meeting on Image Formation in X-Ray Computed Tomography, Salt Lake City, 2018, pp. 293-296.

[18] R. Cierniak , A. Lorent, Comparison of algebraic and analytical approaches to the formulation of the statistical model-based reconstruction problem for X-ray computed tomography, Computerized Medical Imaging and Graphics, vol. 52, 2016, pp. 19-27.

[19] R. Cierniak, P. Pluta, Fast statistical reconstruction algorithm for a CT scanner with flying focal spot, in Proc. of the 6th International Meeting on Image Formation in X-Ray Computed Tomography, Regensburg, 2020, pp.586-589.

[20] R. Cierniak, P. Pluta, Statistical iterative reconstruction algorithm based on a continuous-tocontinuous model formulated for spiral cone-beam CT, In Proc. of the International Conference on Computational Science (ICCS 2020), Amsterdam, 2020, LNCS 12139, pp. 613-620.

[21] J. El Zini, Y. Rizk, M. Awad, An optimized parallel implementation of non-iteratively trained recurrent neural networks, Journal of Artificial Intelligence and Soft Computing Research, vol. 11, 2020, pp. $33-50$.

[22] X. Wang, R.D. MacDougall, C.A. Bouman, S.K. Warfield, Model-based iterative reconstruction for dual source and flying focal spot computed tomography, arXiv:2001.09471v2, 14.II.2020.

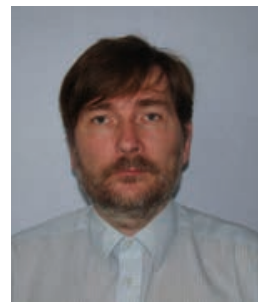

Robert Cierniak received an M.S. degree in Electronics Engineering in 1990, and a Ph.D. degree in Computer Science in 1995, both from Cracow University of Technology, Poland. From 1991 to 2021 he held research at the Czestochowa University of Technology, Poland. He worked from 1991 to 1996 in the Institute of Electronics and Control Systems, and from 1996 to 2009 in the Institute of Computer Engineering, as an assistant professor. He has now become a full professor at this Institute. His main interests include neural networks, biomedical applications of artificial intelligence, computer vision, and image processing.

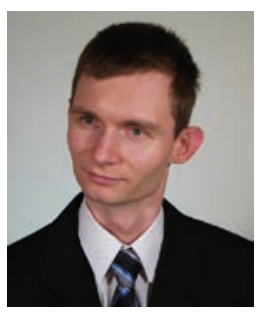

Piotr Pluta graduated from the University of Opole in Computer Science in 2013. He began Ph.D. studies at the Czestochowa University of Technology, at the Faculty of Mechanical Engineering and Computer Science, in 2015. His research activities include optimization and parallelization of algorithms using CUDA/PTX technologies and assembler.
Marek Waligóra earned his MD from the Faculty of Medicine of the Silesian Medical University in Katowice, Poland, in 1991. Dr. Waligóra holds a Ph.D. also from Silesian Medical University in 1997. He joined the Clinical Diagnostic Imaging Department, University of Opole. His main scientific interests and practice involve classic radiology, magnetic resonance imaging, computed tomography.

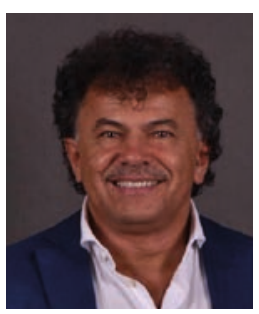

Zdzisław Szymański is a professor at the University of Social Sciences in Łódź, Poland. He received a Ph.D. degree from the Lviv Polytechnic National University in 2008. His research interests include issues related to human capital management, including the perspective of organizational culture and the application of artificial intelligence methods in management. He is the author of over 50 publications. He serves as a Rector's Plenipotentiary for Education Quality. 


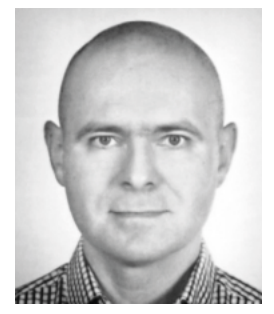

Konrad Grzanek, scientist, programmer and lecturer. Graduate of the Technical University of Łódź (FTIMS). Assistant professor at the Social Academy of Sciences. He holds a Ph.D. from Częstochowa University of Technology (CUT). His research interests focus on programming languages, software quality, software development processes, and artificial intelligence, in particular on combining machine learning methods with static software analysis. As a programmer, he is an advocate and promoter of the functional programming style. Author of over 30 publications related to various problems of computer science and software engineering.

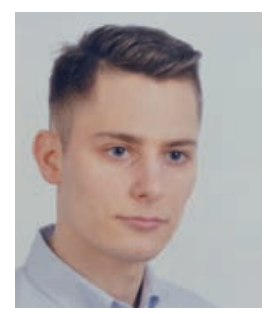

Filip Pałka received his M.Sc. degree in computer science at the Cracow University of Technology, Kraków, Poland, in 2019. Since then, he has been working as a research and teaching assistant at the Department of Computer Science, Faculty of Computer Science and Telecommunications of the Cracow University of Technology. His research focuses mainly on machine learning, deep learning, evolutionary algorithms, specifically on their use for medical data.

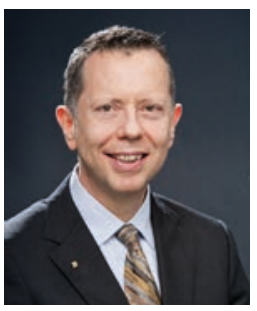

Vincenzo Piuri has received his Ph.D. in computer engineering at Polytechnic of Milan, Italy (1989). He is Full Professor in computer engineering at the University of Milan, Italy (since 2000). He has been Associate Professor at Polytechnic of Milan, Italy and Visiting Professor at the University of Texas at Austin, USA, and visiting researcher at George Mason University, USA.

His main research interests are: artificial intelligence, computational intelligence, intelligent systems, machine learning, pattern analysis and recognition, signal and image processing, biometrics, intelligent measurement systems, industrial applications, digital processing architectures, fault tolerance, cloud computing infrastructures, and internet-ofthings. Original results have been published in $400+$ papers in international journals, proceedings of international conferences, books, and book chapters.

He is Fellow of the IEEE, Distinguished Scientist of ACM, and Senior Member of INNS. He is President of the IEEE Systems Council (2020-21) and IEEE Region 8 Director-elect (2021-22), and has been IEEE Vice President for Technical Activities (2015), IEEE Director, President of the IEEE Computational Intelligence Society, Vice President for Education of the IEEE Biometrics Council, Vice President for Publications of the IEEE Instrumentation and Measurement Society and the IEEE Systems Council, and Vice President for Membership of the IEEE Computational Intelligence Society.

He has been Editor-in-Chief of the IEEE Systems Journal (2013-19). He is Associate Editor of the IEEE Transactions on Cloud Computing and has been Associate Editor of the IEEE Transactions on Computers, the IEEE Transactions on Neural Networks, the IEEE Transactions on Instrumentation and Measurement, and IEEE Access.

He received the IEEE Instrumentation and Measurement Society Technical Award (2002) and the IEEE TAB Hall of Honor (2019). He is Honorary Professor at: Obuda University, Hungary; Guangdong University of Petrochemical Technology, China; Northeastern University, China; Muroran Institute of Technology, Japan; Amity University, India; and Galgotias University, India. 